\title{
Review
}

\section{Bronchoscopic Treatment of Emphysema: State of the Art}

\author{
Stefano Gasparini ${ }^{a}$ Lina Zuccatosta ${ }^{a}$ Martina Bonifazi ${ }^{a}$ Chris T. Bolliger ${ }^{b}$ \\ a Pulmonary Diseases Unit, Department of Immunoallergic and Respiratory Diseases, Azienda

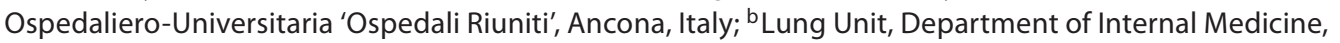 \\ Tygerberg Hospital, Cape Town, South Africa
}

\section{Key Words}

Emphysema Endoscopic lung volume reduction •

Bronchial valves $\cdot$ Lung sealant $\cdot$ Lung coils $\cdot$ Bronchoscopic thermal vapor ablation

\begin{abstract}
In recent years, different bronchoscopic techniques have been proposed for the treatment of emphysema, with the aim of obtaining the same clinical and functional advantages of lung volume reduction surgical techniques while reducing risks and costs. Such techniques can be classified into: methods employing devices that block the airways (e.g. spigots and unidirectional valves), methods that have a direct effect on the lung parenchyma (polymeric lung volume reduction, coils and thermal vapor ablation) and procedures that facilitate the expiration of trapped air from the emphysematous lung (airway bypass). This review aimed to evaluate the indications, outcomes and safety of the different techniques, based on the evidence from the available literature. Results obtained by these methods are encouraging, but they are still based mainly on studies with small groups of patients. However, several trials are ongoing and in the near future we will acquire more knowledge which should lead to a better optimization of these procedures. Mean-
\end{abstract}

\section{KARGER}

Fax +4161306 1234

E-Mail karger@karger.ch

www.karger.com
(C) 2012 S. Karger AG, Basel

0025-7931/12/0843-0250\$38.00/0

Accessible online at:

www.karger.com/res while, the bronchoscopic treatment of emphysema cannot yet be considered a standard of care and patients should be treated in the context of clinical trials or controlled registries, with well-defined programs of evaluation and follow-up.

Copyright $\odot 2012$ S. Karger AG, Basel

\section{Introduction}

The first attempts of surgical lung volume reduction (LVRS) for the treatment of emphysema were performed in the 1950s [1,2], but it was only in 2003, with the publication of the results of the National Emphysema Treatment Trial (NETT) [3] that this procedure demonstrated its ability to improve the clinical and functional status of selected patients affected by emphysema. In the NETT study, a total of 1,218 patients with severe emphysema were randomized to receive LVRS (608 patients) or best medical treatment (610 patients). Subjects with forced expiratory volume in $1 \mathrm{~s}\left(\mathrm{FEV}_{1}\right)$ or with diffusion capacity less than $20 \%$ of predicted, and subjects with homogeneous emphysema were excluded because of a high surgical risk and a low probability of benefitting from the surgery. The results of this trial showed an improvement of exercise capacity of more than $10 \mathrm{~W}$ in 28,22 and $15 \%$ of 
patients, respectively, at 6, 12 and 24 months after surgery in comparison to an improvement in 4, 5 and $3 \%$ of patients in the control arm. The mortality rate in the 90 days after surgery was $7.9 \%$, significantly higher than the $1.3 \%$ for the group treated with medical therapy. However, if the analysis of the results is limited to the subgroup of patients with predominant upper-lobe emphysema and low basal exercise capacity, mortality was lower in the surgical group (2.9\%) than in the control group (3.3\%). This study identified the characteristics of emphysema patients who might benefit from LVRS (subjects with predominant upper-lobe emphysema and low exercise capacity) and receive not only a functional and clinical improvement, but also an increase in survival.

However, some concerns remain regarding LVRS, including its long-term efficacy ( 24 months after the procedure there is a trend of functional parameters to return towards baseline values) [3], safety (prolonged air-leak is described in $30-48 \%$ of cases after LVRS) [4] and costs related not only to the surgical procedure, but also to the long hospital stay (in the NETT study, 28.1\% of patients were still in hospital 1 month after the intervention) [3]. A recent long-term analysis of the NETT study confirmed a 5 -year survival benefit for the surgically treated patients in the subgroup with heterogeneous upper-lobe emphysema (70 vs. $60 \%$ in the medical group) $(\mathrm{p}=0.02)$, whereas this was not the case for the patients with homogeneous emphysema [5]. The considerable morbidity and mortality associated with LVRS prompted reflection on other less invasive ways to achieve LVR, such as endoscopic techniques.

A growing enthusiasm has permeated the world of interventional pulmonology since the beginning of the last decade when the first studies were published on achieving LVR in emphysematous patients via bronchoscopic procedures [6, 7]. The development of bronchoscopic techniques is an attempt to obtain the same results as surgery for the treatment of emphysema, using procedures that are less invasive, potentially reversible and feasible in an outpatient setting at reduced risks and costs. Furthermore, bronchoscopic techniques could also be more suitable for patients who might not be good candidates for surgery, such as patients with predominant lower-lobe emphysema.

In recent years, several new technologies have been introduced, thereby pressuring interventional pulmonologists to treat patients with new devices that have not yet fully been evaluated in terms of their efficacy and safety. Indications, outcomes, contraindications, patient-selection criteria and the costs of these new treatment modal-
Table 1. Techniques for the bronchoscopic treatment of emphysema

Bronchial blocking devices

Spigots (Endobronchial Watanabe Spigots)

Unidirectional valves (Zephyr, IBV or Novatech)

Devices that work at the pulmonary parenchyma level

Sealant (AeriSeal)

Coils (PneumRx)

Vapor (InterVapor)

Extra-anatomical airways

Airway bypass (Exhale System)

ities have not been well defined. Several studies are ongoing and new results continue to appear in the literature. The topic is fascinating and it seems interesting and timely to attempt to answer, on the basis of the available literature results, the following questions. (1) What is the current state of the bronchoscopic management of emphysema? (2) What are the technical characteristics, the advantages and limits of each procedure? (3) What are the implications for the daily clinical practice?

Table 1 shows the different bronchoscopic techniques that have been proposed, classified on the basis of the underlying mechanism into 3 main groups: blocking devices that act at proximal bronchi level with the aim to produce bronchial occlusion and atelectasis, devices that work at the pulmonary parenchymal level and methods that create extra-anatomical airways to facilitate lung deflation.

\section{Bronchial Blocking Devices}

\section{Plugs}

The only bronchial plugs available on the market today are the so-called 'Watanabe Spigots', named after the Japanese pulmonologist who proposed them [8]. They are made of silicon and have a truncated conical shape and lateral studs that facilitate anchorage to the bronchial wall. Watanabe Spigots (Novatech, La Ciotat, France) are available in 3 different sizes $(5,6$ and $7 \mathrm{~mm}$ ) (fig. 1a). In effect, such devices were initially introduced into the clinical practice for the treatment of pulmonary fistula and persistent pneumothorax with continuous air leakage. They have also been used to achieve LVR in cases of emphysema, but the results, limited to a small number of patients, have only been published in abstract form [911]. In a study by Miyazawa [10], out of 7 patients who 
were treated, 5 obtained an improvement in quality of life, vital capacity (from 2.54 to 2.82 liters) and exercise capacity [from 260 to $317 \mathrm{~m}$ with the 6-minute walking test $(6 \mathrm{mWT})]$. Pneumonia as a complication of this procedure was described in 5 cases out of 40 treated in 3 studies (12.5\%). This high rate may be as a consequence of the inability of secretions to drain past obstructed bronchi. A multicenter study using Watanabe spigots to treat emphysema is ongoing in Japan, but results are not yet available [11].

\section{Unidirectional Endobronchial Valves}

Unidirectional endobronchial valves are certainly the most widely studied devices for the bronchoscopic treatment of emphysema and have the largest series of treated patients.

The characteristic feature of the endobronchial valves is the ability to block the entrance of air during inspiration, while permitting the emission of air and secretions during expiration.

Two different types of valves are available on the market: Zephyr valves (Pulmonx, Inc., Palo Alto, Calif., USA) and IBV valves (Spiration Inc., Redmond, Wash., USA). Both devices are self-expanding and retained into a catheter that can be introduced through the working channel of a flexible bronchoscope.

Zephyr valves (fig. 1b) are made of a nitinol mesh covered by silicon, with a double silicon membrane inside that opens during expiration and closes during inspiration, a mechanism similar to Heimlich valves used for pleural drainage. There are 2 sizes of Zephyr valves available, 1 small $(4.0-7.0 \mathrm{~mm})$ for segmental bronchi and 1 larger $(5.5-8.5 \mathrm{~mm})$ for lobar bronchi. Anchorage of the valve to the bronchial wall is achieved via the self-expanding strength and irregular surface of the nitinol mesh. Following the first pilot studies on a small number of patients $[12,13]$ documenting the safety and the feasibility of the procedure, a multicenter trial on 98 patients affected by severe emphysema was conducted and the results published in 2006 [14]. Ninety days after the treatment, there was a significant increase of $\mathrm{FEV}_{1}(+10.7 \%)$ and of vital capacity (+9.0\%), a reduction of residual volume (RV) $(-4.9 \%)$ and an improvement of exercise capacity $(+23 \%$ at $6 \mathrm{mWT})$. Better results were obtained in subjects who underwent lobar exclusion than those who received a segmental treatment. Severe complications were: 1 death (a patient that already underwent a prior right upper lobectomy for cancer), 3 pneumothoraces and 4 prolonged air leaks. Evident from this study is that the response to treatment was variable and that functional
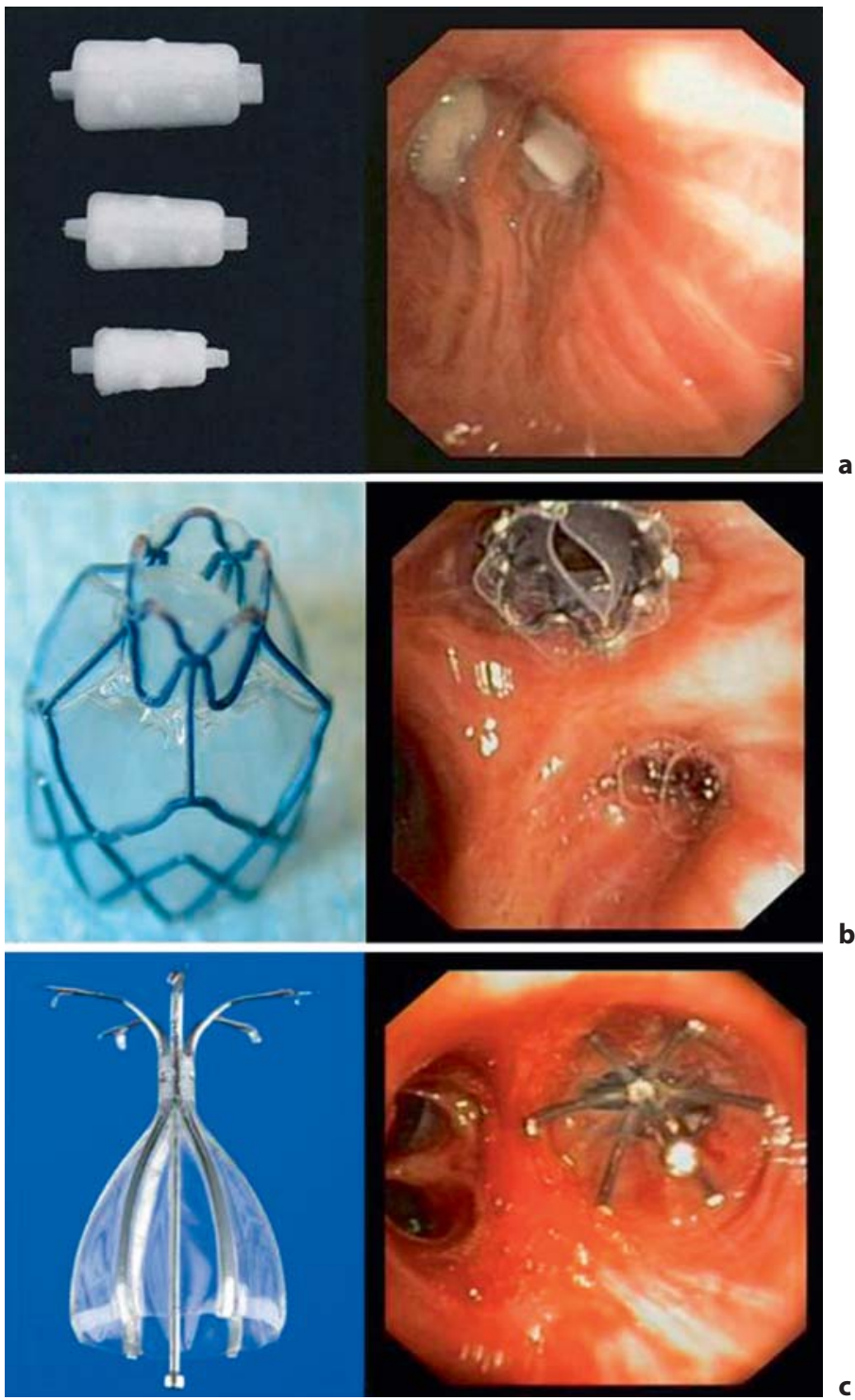

Fig. 1. Bronchial blocking devices for bronchoscopic treatment of emphysema. a Watanabe Spigots. b Unidirectional Zephyr valve. c Unidirectional IBV valve.

benefit was more frequent in patients who developed anatomic atelectasis. Lobar bronchus occlusion does not guarantee atelectasis in all patients, possibly due to the presence of interlobar collateral ventilation. This condition, that has been described even in normal subjects and may be more frequent in emphysematous patients $[15,16]$, allows air to enter the treated lobe through pores present at the interlobar fissure level, potentially negating the efficacy of the valves. 
To evaluate the effectiveness and the safety of Zephyr valves for the treatment of emphysema, an international, multicenter, prospective, randomized study (Endobronchial Valve for Emphysema Palliation Trial; VENT) was performed [17]. The trial was conducted at 31 centers in the USA and at 23 sites in Europe with a total number of 492 randomized patients (321 in USA and 171 in Europe). After a period of 6-8 weeks in a rehabilitation program, patients were randomly assigned (2:1) to the valve-treatment arm or the control arm (best standard medical care). The results of the US and European cohorts were evaluated and published separately in two different papers $[18,19]$.

In the US study [18], after 6 months, the endobronchial valve treatment had induced a modest improvement in lung function $\left(\mathrm{FEV}_{1}=+4.3 \%\right)$ and exercise tolerance $(6 \mathrm{mWT}=+2.5 \%)$ and a small change in patients' quality of life, as evaluated by the St. George Respiratory Questionnaire (SGRQ) ( -2.8 points). Even if these changes were statistically significant in comparison to the control group, they are hardly of clinical relevance. However, if the analysis were limited to patients with a higher CT scan evidence of emphysema heterogeneity, there was a greater improvement in both the $\mathrm{FEV}_{1}(+10.7 \%)$ and the $6 \mathrm{mWT}(+12.4 \%)$. The other characteristic that predicted a better response to treatment was the fissure integrity as evaluated by CT scan, ostensibly because the patients with evidence of a complete fissure have a lower probability for collateral ventilation. The subgroup of patients with fissure integrity showed an improvement of $16.2 \%$ for $\mathrm{FEV}_{1}$ and $7.7 \%$ for $6 \mathrm{mWT}$.

In the European study [19], the global results were similar, showing a modest benefit in the valve-treatment arm after 6 months in comparison to the control group for $\mathrm{FEV}_{1}$ (+7 vs. $0.5 \%$ ), cycle ergometry workload (+2 vs. $-3 \mathrm{~W}$ ) and SGRQ (-5 vs. 0.3 points). Also in the European cohort, fissure integrity, present in about one third of the patients, was a good indicator for a better response $\left(\mathrm{FEV}_{1}=+16 \%\right.$; cycle workload $\left.=+4 \mathrm{~W} ; 6 \mathrm{mWT}=+11 \%\right)$.

Another factor evaluated in the European study is the CT evidence of complete lobar occlusion provided by the valve placement. Incomplete occlusion provides a conduit for air to enter upon inspiration and this was identified by the presence of air between the valve and the bronchial wall, suggesting a leak. CT evidence of lobar occlusion was found in less than half of the valve-treated patients and in this subgroup the clinical outcomes were better than subjects with incomplete occlusion $\left[\mathrm{FEV}_{1}=\right.$ +26 vs. $+6 \%$; cycle ergometry workload $=+8$ vs. $0 \%$; $6 \mathrm{mWT}=+22$ vs. $-2 \%$; SGRQ $=-10$ vs. $-2 ; \mathrm{RV} /$ total lung capacity $(\mathrm{TLC})=-14$ vs. $-1 \%]$.

Bronchoscopic Treatment of Emphysema
Both reports of the VENT study demonstrated the safety of the valve treatment, showing a small incidence of complications. In the US study [18], the most common complication was pneumonia distal to the valves $(4.2 \%$ at 12 months, resolved in 6 patients without valve removal and in 6 patients after valve removal). Other complications were hemoptysis $(5.6 \%$ in the first 6 months after treatment and 6.1\% 6-12 months after treatment) and pneumothorax (4.2\%). Exacerbations of COPD were more frequent in the valve-treated group than in the control arm in the first 6 months (7.9 vs. 1.1\%), but occurred at a similar rate during the period 6-12 months after. In the European study [19], the incidence of pneumothorax was $4.5 \%$, pneumonia distal to the valve $3.6 \%$ and hemoptysis $5.4 \%$. Occurrence of COPD exacerbations did not differ significantly between the treated patients and the control group.

These studies demonstrate the strong influence of anatomic characteristics on valve treatment outcomes, such as fissure integrity and emphysema heterogeneity and the importance of technical factors such as the achievement of a complete lobar occlusion, underscoring the importance of both optimal procedural technique and careful patient selection.

The second model of valve available on the market is the IBV valve, an umbrella-shaped device made by a nitinol mesh covered by a polyurethane membrane (fig. 1c). The valve is secured to the bronchial wall by 5 hook-like anchors and can be removed by grasping and pulling on its proximal central rod with forceps. It is available in 3 different sizes (5, 6 and $7 \mathrm{~mm}$ ).

A pilot multicenter study with IBV valves was performed in the US on 91 patients affected by heterogeneous predominant upper-lobe emphysema [20]. It is interesting to note that in this trial, following the observation of a higher incidence of pneumothorax occurring with complete lobar occlusion (especially of the left upper lobe), the therapeutic strategy was modified during the study. For this reason, the trial was carried on with the bilateral treatment of both upper lobes, keeping open the lingula and avoiding complete lobar occlusion. The aim of this treatment strategy, which is not dependent on lobar atelectasis, is to shift the ventilation to the untreated healthier lobes, improving ventilation/perfusion matching and to reduce dynamic hyperinflation. The results of this trial showed no modification of functional parameters $\left(\mathrm{FEV}_{1}\right.$, total lung volume and exercise tests were unchanged) but a significant improvement of quality of life (SGRQ $=-8.2$ points at 6 months). The same modality of treatment was evaluated in a prospective, randomized,

Respiration 2012;84:250-263 
multicenter, sham-controlled European study [21] carried out on 73 patients with predominant upper-lobe emphysema. Thirty-seven patients were randomized to receive valve treatment (implantation of IBV valves in the bilateral upper lobes, without complete lobar occlusion) and 36 patients underwent bronchoscopy with a sham procedure. No functional improvement was observed in the treatment group of this study either; however, a volume reduction evaluated by CT scan and a significant improvement of the quality of life in comparison to the control group were reported. In particular, 8 out of 33 (24\%) in the treatment group exceeded the minimum threshold changes for CT lung volumes and SGRQ total score, compared with no subjects (out of 35 ) in the control group [21].

The strategy to keep open a segmental bronchus has been questioned by a study published by Eberhardt et al. [22]. Twenty-two patients were randomized to receive complete unilateral occlusion or bilateral upper-lobe treatment with incomplete occlusion. The functional improvement was significantly greater in the group that underwent a complete lobar treatment $\left(\mathrm{FEV}_{1}=+21.4 \mathrm{vs}\right.$. $-0.03 \%$ ), with just 1 case of pneumothorax in this group. This study demonstrated that the unilateral procedure aimed at obtaining complete lobar occlusion is more effective than the bilateral incomplete treatment and that, given the superior outcome, the increased risk of pneumothorax may be acceptable.

More recently, a third valve model has been proposed (Endobronchial Miyazawa Valve, Novatech, La Ciotat, France). The Miyazawa valve is a silicon device covered with small studs to prevent migration and a duckbill mechanism that permits exhalation of air from the distal lung segment but does not allow reinflation. The first study with the Miyazawa valve [23] on 12 patients affected by advanced emphysema revealed a reduction of volume in the treated lobe $(-17.7 \%$ at 1 month and $-12 \%$ at 6 months) and an improvement of quality of life and exercise capacity $(6 \mathrm{mWT}=+47 \%$ at 1 month and $+57 \%$ at 6 months). Another positive experience with this valve was published as a case report [24], but studies on large numbers of patients are still lacking.

Our experience with the use of unidirectional valves for the treatment of emphysema, based on the patients treated at the Azienda Ospedaliero-Universitaria 'Ospedali Riuniti', Ancona (Italy), is summarized in figure 2. From 2005 to August 2011 we treated 34 patients affected by heterogeneous emphysema. The treatment was performed unilaterally with the aim of obtaining complete lobar occlusion. Thirty-two patients were treated on

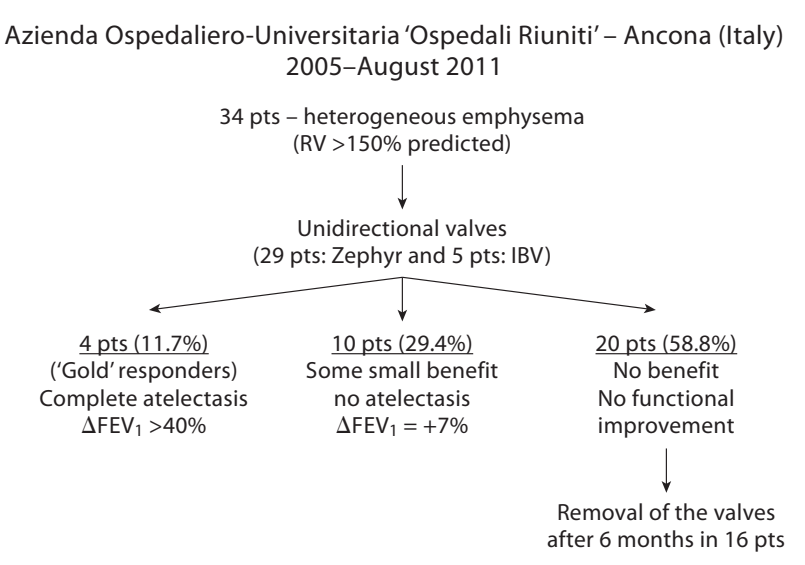

Fig. 2. Experience from the use of unidirectional valves for the treatment of emphysema, based on the patients treated at the Azienda Ospedaliero-Universitaria, Ospedale Riuniti, Ancona, Italy. pts $=$ Patients.

the upper lobe and 2 patients on the lower lobe. Four patients $(11.7 \%)$ benefited greatly, with a functional $\left(\mathrm{FEV}_{1}\right.$ increase of more than 40\%) and clinically relevant improvement. All these patients showed an atelectasis of the treated lobe. In 10 patients (29.4\%), we observed some small benefit $\left(\mathrm{FEV}_{1}=+7 \%\right)$, but atelectasis was not present. In 20 patients (58.8\%), there were neither functional nor clinical improvements and the valves were removed in 16 patients after 6 months. It must be observed that some patients in our series were treated before the awareness that fissure integrity is an important predictive factor for the outcome, so that some were not evaluated for this condition. This factor could potentially explain the high incidence of nonresponders.

The collective experience chronicled above allows us to draw some reasonable conclusions on the use of unidirectional valves, as summarized below.

(1) There is no comparative study demonstrating the advantages of one model of valve over another.

(2) Valves can also be used for predominant lower-lobe emphysema (fig. 3).

(3) The best clinical and functional results seem to be correlated with the development of atelectasis, and therefore a true volume reduction - without atelectasis, the improvement is generally modest or absent.

(4) Atelectasis occurs in a minority of patients. The main reason why valves do not work in some patients is the presence of collateral ventilation. Another important factor could be the technical failure of the valves to accomplish a complete occlusion of the target bronchus. 


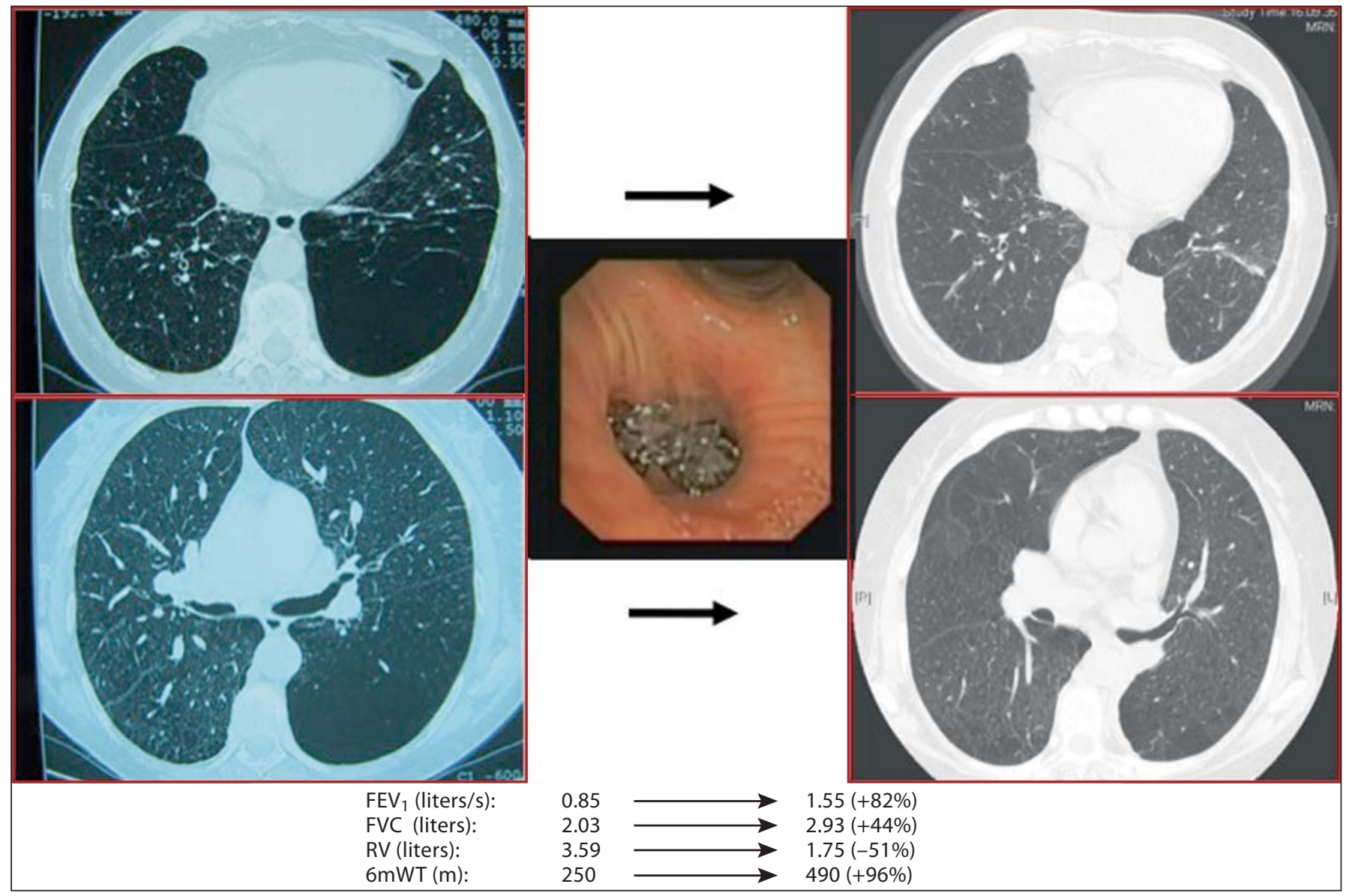

Fig. 3. A case of a 62-year-old woman with predominant left lower-lobe emphysema. Left: CT scan before the procedure. Two Zephyr valves were positioned (1 in the left segment No. 6 and the other at the level of the orifice of the left lower lobe). Right: CT scan 30 days after the procedure shows a complete atelectasis of the left lower lobe and an evident volume reduction of the left hemithorax. Functional evaluation before and after the procedure shows a significant improvement of all the parameters.

(5) The evaluation of collateral ventilation is a crucial step in selecting patients that might benefit from valve treatment. The interlobar fissure integrity evaluated by CT scan seems to be a good predictor of the absence of collateral ventilation. The assessment of collateral ventilation can also be performed with the use of an endobronchial catheter system (Chartis System, Pulmonx Inc., Redwood, Calif., USA) that can be inserted through a flexible bronchoscope [25]. The Chartis catheter has at its tip a balloon that after inflation, blocks the air entrance into the target bronchus. The catheter is connected to an external console that detects the flow and pressure of air coming from the balloon-occluded bronchus. When airflow from the target lobe trends over time towards zero, collateral ventilation is assumed to be lim- ited. On the contrary, when airflow from the target lobe persists, significant cross-communication between lobes may be present. In a study performed on 20 patients, the resistance measurements assessed by Chartis correlated with post-implantation atelectasis in $90 \%$ of cases [26].

(6) The incidence of complications related to the procedure is quite low and valve implantation can be considered safe. Pneumonia (3.6-4.2\%), pneumothorax (4.24.5\%), hemoptysis (5.4-6.1\%) and exacerbation of COPD (7.9\%) are the most frequent complications. However, one potential advantage of valves is their easy removability, even a long time after implantation. 
Fig. 4. The AeriSeal System. a, b Synthetic polymeric substance and cross-linker that must be mixed before instillation through different syringes to initiate polymerization. c The foam sealant is delivered in a subsegmental bronchus through a catheter with its tip positioned $2 \mathrm{~cm}$ beyond the bronchoscope. $\mathbf{d}$ The foam sealant fills the subsegmental bronchus after delivering.
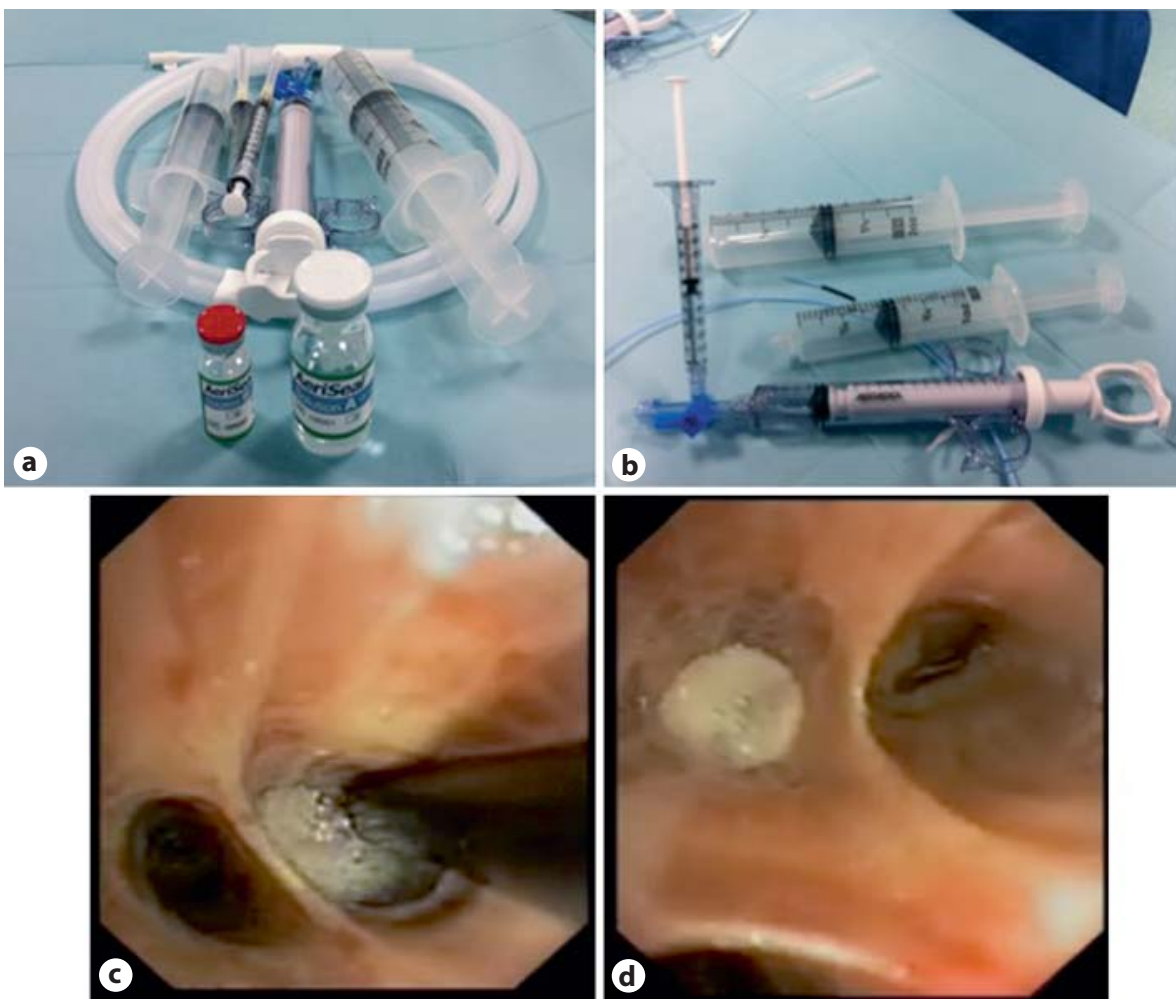

\section{Devices That Work at the Pulmonary Parenchymal Level}

\section{Sealant}

First-generation products used in bronchoscopic LVR were biological substances (so-called 'biological lung volume reduction') aimed at obtaining atelectasis and subsequent fibrosis of the lung parenchyma. After the first pilot studies in animal models and emphysematous patients [27-29] had demonstrated the safety of the technique and the efficacy in inducing LVR, a large multicenter phase- 2 dose-ranging trial was conducted in 50 patients affected by heterogeneous predominant upperlobe emphysema [30]. The treatment consisted of bronchoscopic instillation of a fibrinogen biopharmaceutical suspension and thrombin solution that polymerized in situ to form a hydrogel able to initiate a localized inflammatory reaction that collapsed the lung region over 4-6 weeks. Twenty-eight patients were treated at 8 subsegmental sites (4 in each lung) with low-dose (LD) hydrogel $(10 \mathrm{ml})$ and 22 with high-dose (HD) hydrogel $(20 \mathrm{ml})$ per subsegment. Fourteen patients in the LD group and 10 in the HD group received 2 treatment sessions separated by 6-12 weeks, while 14 in the LD and 12 in the HD group underwent a single session. The results of this trial showed a reduction in the ratio RV/TLC at 12 weeks in both the LD (-6.4\%) and HD (-5.5\%) groups. At 3 months, FEV had improved by $9.9 \%$ in the LD group and by $17.7 \%$ in the HD-treated patients. There was also a significant improvement in forced vital capacity (FVC) $(+9.8 \%$ for LD and 11.9 for HD patients) and in exercise capacity $(6 \mathrm{mWT}=+38.6$ and $+6.4 \%$, respectively, in the LD and HD groups). Six months after treatment, all the functional, measured parameters remained significantly higher than baseline for the HD group, while in the LD group the values of RV, RV/TLC and FVC did not maintain a significant difference with respect to baseline.

First-generation biological substances have now been replaced by synthetic polymeric foam (Aeris Therapeutics, Woburn, Mass., USA), administered to the subsegmental bronchi. The foam flows into the peripheral airways and acts as a glue that seals the target regions and produces consequent airway collapse and atelectasis (fig. $4,5)$. In a multicenter study conducted on 25 patients with advanced heterogeneous emphysema in Germany [31], synthetic polymer sealant was instilled initially at 2-4 subsegments. After 12 weeks, patients were eligible for repeat treatment at a total of 6 sites. After 24 weeks, 
Fig. 5. CT scan of a 67-year-old patient treated with the AeriSeal System on the left upper lobe before (left) and 1 month after the procedure (right). Functional parameters before and after the treatment are reported. Three months later, the patient was treated on the right side with further functional improvement.

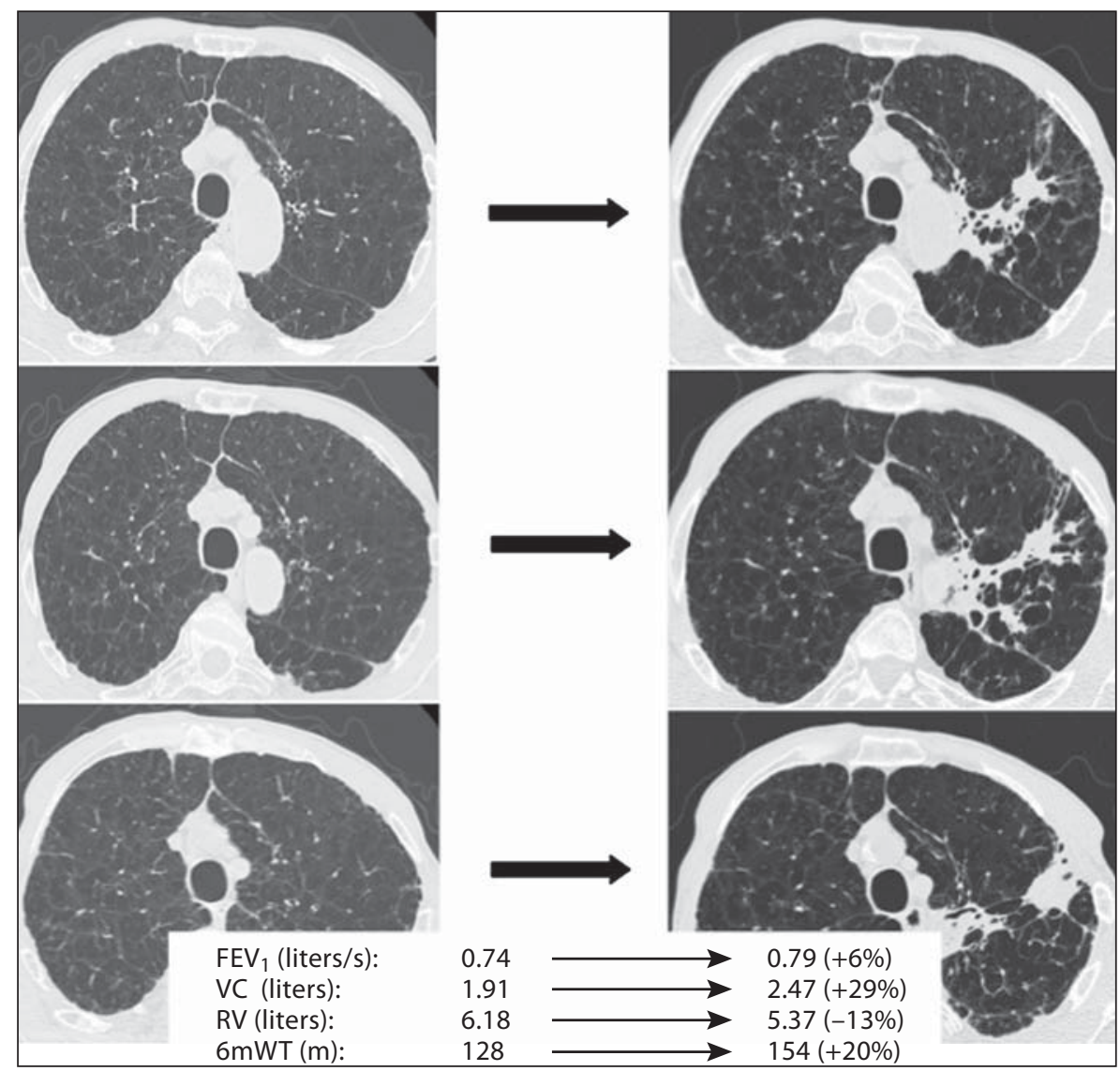

there was an improvement in $\mathrm{FEV}_{1}(+10.0 \pm 19.8 \%), \mathrm{FVC}$ $(+15.8 \pm 22.2 \%)$ and $6 \mathrm{mWT}(+24.6 \pm 58.9 \mathrm{~m})$, while RV/TLC decreased ( $-4.7 \pm 9.5 \%)$, but only the improvement in FVC was statistically significant. Results were better in the 14 GOLD-stage-III patients $\left(\mathrm{FEV}_{1}=+15.9\right.$ $\pm 22.6 \% ; \mathrm{FVC}=+24.1 \pm 22.7 \% ; \mathrm{RV} / \mathrm{TLC}=-7.4 \pm$ $10.3 \% ; 6 \mathrm{mWT}=+28.7 \pm 59.6 \mathrm{~m}$ ) in comparison to the 11 GOLD-stage-IV subjects for whom the benefit was less relevant $\left(\mathrm{FEV}_{1}=+2.3 \pm 12.3 \% ; \mathrm{FVC}=+2.6 \pm 21.1 \%\right.$; RV/ TLC $=-0.5 \pm 6.4 \% ; 6 \mathrm{mWT}=+28.3 \pm 58.4 \mathrm{~m}$ ). There were no serious procedural or immediate postprocedural complications and no treatment-related deaths. The treatment was associated with a 'flu-like' reaction with elevated inflammatory markers, dyspnea, fever and leukocy tosis. On chest radiograph, 16 patients had infiltrates and 12 had chest pain. These symptoms were generally self-limited and resolved within 24-96 h. COPD exacerbations occurred in 6 GOLD-stage-III patients and in 4 GOLD-stage-IV patients in the period after treatment. These study results are promising, but the small number of patients makes it necessary to conduct additional tri- als, to be able to draw final conclusions about the safety and effectiveness of polymeric sealant.

Based on the above evidence regarding polymeric lung sealant, we present the following considerations.

(1) This treatment acts at the alveolar rather than the airway level and for this reason should be not influenced by collateral ventilation.

(2) The procedure appears easy to perform, but, in contrast to endobronchial valves, it is not reversible. Optimal patient and target site selection is therefore crucial.

(3) The procedure is not indicated if there are large bullae $(>5 \mathrm{~cm})$ and for predominant lower-lobe emphysema.

(4) The literature concerns a small number of patients and further studies on larger populations are required. A multicenter, international, controlled, phase-IV, randomized study (ASPIRE trial) is currently ongoing to provide more data on this kind of treatment [32].

(5) The applicability of this technique to patients with homogeneous emphysema is also under evaluation in pilot studies, but the results are not yet available. 


\section{Coils}

Coils (PneumRx Inc., Mountain View, Calif., USA) are nitinol devices designed to behave as spring elements capable of retracting lung parenchyma and consequently reducing volume, restoring lung tissue tension and restoring radial suspension of the peripheral airways (fig. 6). The preformed nitinol wire coils are bronchoscopically inserted, in an elongated straightened position into subsegmental airways, out into the lung periphery, recovering their predetermined coil shape upon deployment. The insertion process requires first the advance of a guidewire into the selected airway up to $15 \mathrm{~mm}$ from the pleura surface. A catheter is then inserted over the guidewire and the straightened coil is pushed through the catheter under fluoroscopic guidance. After removal of the catheter, the coil recovers its original shape and bundles up the surrounding lung parenchyma. The coils are made in a range of lengths $(70-200 \mathrm{~mm})$ and on average, 10 coils per treated lobe are deployed (fig. 7).

A pilot study involving 8 animals and 2 human isolated lungs each implanted with 6 coils, demonstrated an average volume reduction of $466 \mathrm{ml}$ [33]. Preliminary studies on patients were mainly designed to assess the safety of the procedure. In the first 11 patients treated with coils, no cases of death, pneumonia or pneumothorax were recorded [34]. Adverse events were: an increase in dyspnea (6 cases), cough (5 cases), exacerbation of COPD (3 cases) and thoracic pain (1 case). Efficacy data showed meaningful improvements only in patients affected by heterogeneous emphysema while there were no significant improvements in patients with homogeneous emphysema [35].

Recently, the results of coil implant in 16 patients with severe heterogeneous emphysema were published [36]. Twelve patients were treated bilaterally in two sequential procedures and 4 patients received coils in one lung. Two hundred and sixty coils were implanted (a median of 10 per procedure). Six months after the procedure, there was a significant improvement in $\mathrm{FEV}_{1}$ $(+14.9 \%), \mathrm{FVC}(+13.4 \%)$ and exercise capacity $(+84.4 \mathrm{~m}$ at $6 \mathrm{mWT}$ ), while $\mathrm{RV}$ was reduced $(-11.4 \%)$. A significant improvement in quality of life evaluated with the SGRQ was also reported (-14.9 points). No life-threatening complications related to coil implant occurred. The adverse events were pneumothorax $1 \mathrm{~h}$ after the procedure ( 1 case), mild hemoptysis in $75 \%$ of the procedures (spontaneously resolved in all cases), and transient chest pain in 4 cases.

The published data on coils supports the following tentative conclusions.

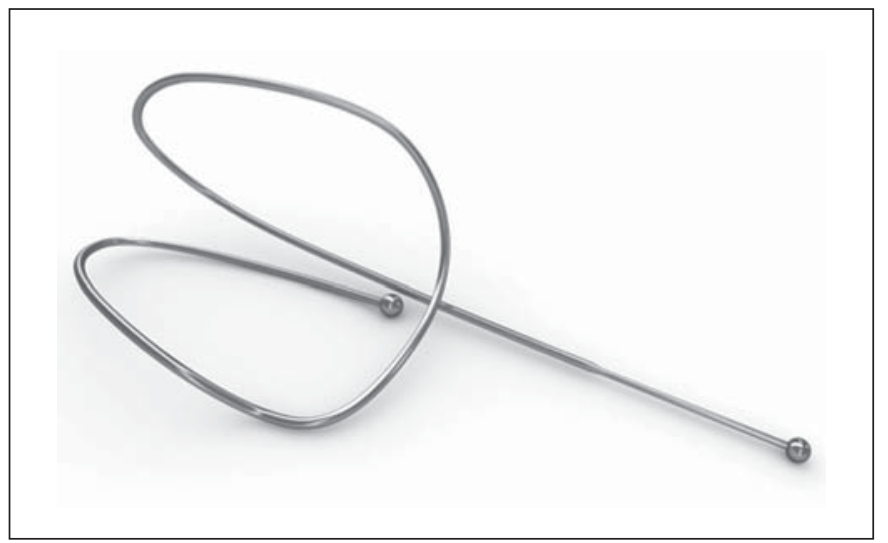

Fig. 6. Coil for the bronchoscopic treatment of emphysema in its predetermined shape which it recovers after deployment into the airways.

(1) The coils seem to confer benefit to patients with heterogeneous emphysema, independent of collateral ventilation.

(2) It is uncertain that this device can be removed long after its deployment.

(3) The coils require an incompletely defined minimal amount of tissue for optimal performance, and for this reason their use is not indicated if the lung parenchyma is too destroyed or if there are large bullae.

(4) The number of treated patients is still small and larger studies are warranted to better define the efficacy and safety profile of this device. Recently, PneumRx received FDA approval to commence a pivotal clinical trial on more than 300 patients in the USA.

\section{Thermal Vapor Ablation}

Bronchoscopic thermal vapor ablation (InterVapor Uptake Medical, Seattle, Wash., USA) is a technique that uses high-temperature water vapor delivered into the target lung segments through a catheter at a precise amount of energy (calories/gram of lung tissue). The heated vapor induces thermal damage and an inflammatory reaction that is followed by permanent fibrosis. In addition to cellular responses to heat damage, the blood flow reduction inducing ischemia may play a large role in determining LVR with this technique [37].

The efficacy of bronchoscopic thermal vapor ablation was demonstrated in animal studies with normal lungs or with papain-induced emphysema. The volume of target areas was reduced by up to $80 \%$ and the volume reduction entity was proportional to the dose of administered vapor [38]. 
Fig. 7. Chest X-ray of a 67-year-old patient treated with 8 coils on the left upper lobe. Functional data before the treatment and 1 month later are reported. This patient was previously treated with a valve implant without any benefit, and so the valves were removed.

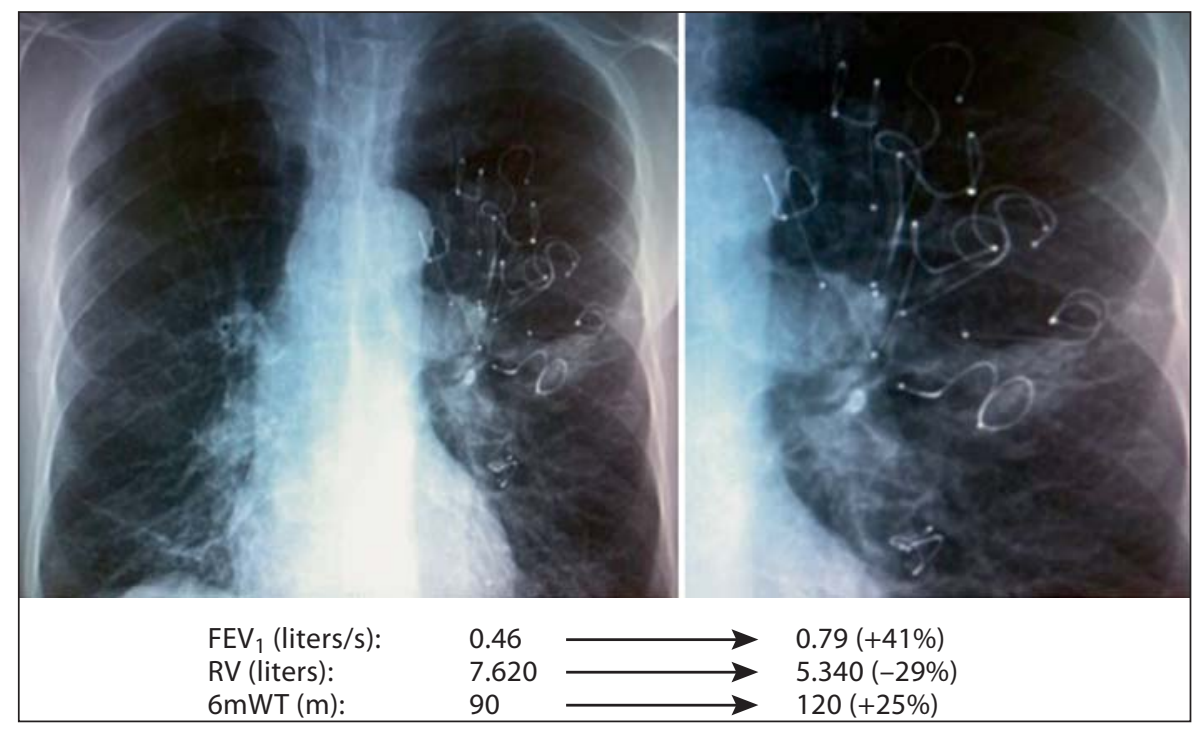

The first feasibility study in humans was performed in 11 patients with severe heterogeneous emphysema who underwent unilateral upper-lobe bronchoscopic application of vapor thermal energy at a low dose $(5 \mathrm{cal} / \mathrm{g}$ of lung tissue) [39]. Efficacy results at 6 months were modest and showed no changes in $\mathrm{FEV}_{1}$ or RV, but there was an improvement in diffusion capacity $(+16 \%)$, in dyspnea score and in quality of life evaluated with the SGRQ (from 64.4 to 49.1 points). Serious adverse complications included 5 cases of probable bacterial pneumonia and 2 cases of COPD exacerbation.

In a following larger study on 44 patients with heterogeneous upper-lobe emphysema, a higher dose of vapor was administered (10 cal/g of lung tissue) [40]. A total of 72 and 58 segments were treated in the right upper lobe $(\mathrm{n}=24)$ and left upper lobe $(\mathrm{n}=20)$, respectively. At 6 months, there was a significant improvement in $\mathrm{FEV}_{1}$ $(+140.8 \pm 26.3 \mathrm{ml})$ and FVC $(+271.0 \pm 71.9 \mathrm{ml})$ and a reduction in RV $(-406.0 \pm 112.9)$. There was also a significant improvement in quality of life, dyspnea index and exercise capacity $(6 \mathrm{mWT}=+46.5 \pm 15.0$ meters $)$. After 6 months, the HRCT measurement of lobar volume was reduced by $48 \%$. Lobar fissure integrity had no or minimal influence on LVR and improvements in clinical outcome [41]. The major total adverse events observed were: COPD exacerbations (10 cases), pneumonia [6], respiratory tract infections [5] and hemoptysis [3]. All the adverse events resolved with medical therapy, except for 1 patient who died secondary to a COPD exacerbation that occurred 67 days after treatment.

Bronchoscopic Treatment of Emphysema

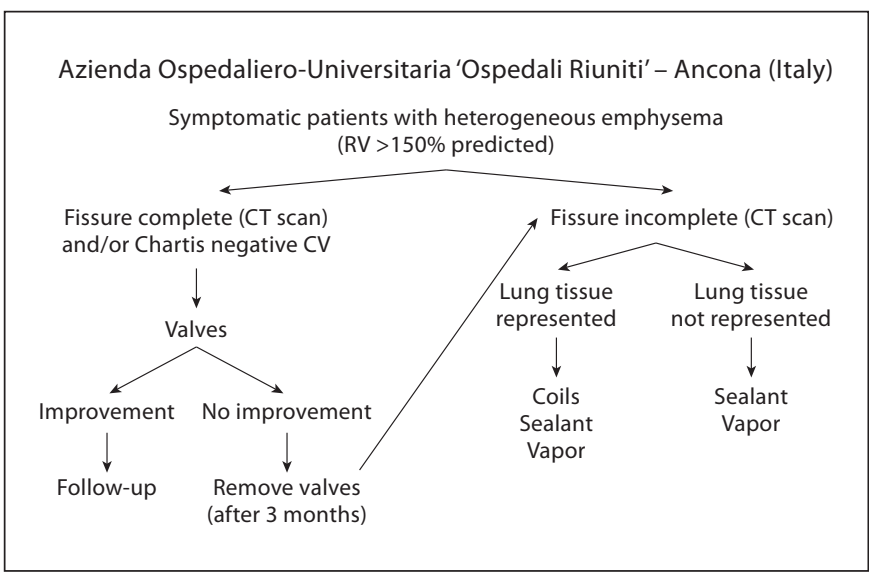

Fig. 8. Flow chart for the bronchoscopic treatment of emphysema followed at the Pulmonary Diseases Unit of the Azienda Ospedaliero-Universitaria 'Ospedali Riuniti', Ancona. Vapor is not yet available in Italy.

For vapor thermal ablation, many of the same considerations discussed above for sealant apply and are presented here.

(1) The treatment is not influenced by collateral ventilation.

(2) The procedure is not reversible.

(3) The technique was utilized only in patients with heterogeneous predominant upper-lobe emphysema and no data are available for predominant lower-lobe emphysema or for patients with homogeneous emphysema. 
(4) Results are available only in a small number of patients and further studies in larger populations are required.

\section{Airway Bypass}

The technique of airway bypass (Exhale Emphysema Treatment System, Broncus Technologies Inc., Mountain View, Calif., USA) is based on the creation of extra-anatomic passages between the hyperinflated lung parenchyma and larger airways, with the aim of facilitating expiration and to decrease air trapping.

The system is based on different components: (1) a Doppler flexible probe with an ultrasonic transducer at its tip, to identify blood vessel-free areas at the level of segmental bronchi, (2) a 25-gauge needle that is used to perforate the bronchial wall, associated with a $2.5-\mathrm{mm}$ dilation balloon, (3) a delivery catheter that is used to place in the hole a paclitaxel-coated stent (length $2 \mathrm{~mm}$, inner diameter $3.3 \mathrm{~mm}$ and outer diameter $5.3 \mathrm{~mm}$ ), designed to reduce granulation and to hold the passage open.

Preliminary pilot studies, on excised lungs and on patients who were already scheduled to undergo lobectomy or lung transplantation, demonstrated the safety of the technique and showed an improvement in functional parameters after the creation of bypasses [42-44].

In a multicenter study of 35 patients [45], 33 with homogeneous emphysema characterised by severe hyperinflation (RV $>220 \%$ predicted), a total of 264 stents were implanted (median 8 stents per patient and range 2-12) [43]. One month after the procedure, the results showed a significant reduction in RV $(-12.4 \%)$ and an improvement in $\mathrm{FEV}_{1}(+7.3 \%)$, vital capacity $(+17.2 \%)$ and $6 \mathrm{mWT}$ $(+37.2 \%)$. At 6 months, there was a trend for the functional parameters to return towards baseline values and only changes in RV and in dyspnea index remained statistically different from the baseline.

The best short-term and long-term results were observed in patients with a high degree of hyperinflation (RV/TLC >0.67). In this study, a death from massive hemoptysis was observed. This adverse event led the authors to recommend that a standby balloon blocker be placed into the main bronchus during the procedure, and to repeat the Doppler scanning after the creation of the hole and before the placement of the stent.

Recently, the results of a randomized, double-blind, sham-controlled study (EASE trial) on 315 patients with emphysema and severe hyperinflation $($ RV/TLC $>0.67)$ were published [46]. Two hundred and eight patients were treated with airway bypass and 107 control patients underwent sham procedures. An immediate improvement was observed after the procedure, but at 6 months no differences were seen between the treatment arm and the control group. The authors concluded that airway bypass is unable to provide a long-term sustainable benefit in patients with severe homogeneous emphysema.

As a consequence of this study, Broncus Technologies Inc. is now exploring new ways to extend the procedure benefit, but at the moment the procedure has been abandoned and there are currently no clinical trials underway with airway bypass [47].

\section{Conclusions}

Within the last decade, the systems for bronchoscopic emphysema treatment have roused great interest among pulmonologists, becoming one of the most exciting technological innovations in the field of bronchoscopy. Even though the results are promising (the functional improvements are greater than those obtained in any pharmacological trial using bronchodilator and anti-inflammatory drugs), the indications and the real long-term efficacy and safety outcomes have not yet been well defined.

Table 2 summarizes the effects of different procedures on functional parameters.

A direct comparison between different techniques is not feasible given the heterogeneity of the study populations and the small sample size of most of the trials.

Since the effects of these bronchoscopic techniques may be more than LVR, as other potential mechanisms may be involved (redistribution of air flow, restoring of lung tissue tension and influence on ventilation/perfusion relationship), it seems that 'bronchoscopic treatment of emphysema' instead of 'bronchoscopic lung volume reduction' would be a more appropriate descriptive term.

The emerging scenario is characterized by the definition of different emphysema phenotypes, since not all the procedures are indicated in all the cases and each technique appears to provide greater benefit to specific subgroups of patients. The assessments of collateral ventilation, emphysema heterogeneity and distribution, the degree of hyperinflation and lung tissue consistency are all elements that must be carefully considered to identify the best technique for each individual patient.

Patient selection, therefore, is key to a successful treatment and close cooperation between bronchoscopists, 
Table 2. Results obtained with the different techniques on functional parameters, exercise capacity and quality of life in the most representative studies published in the literature

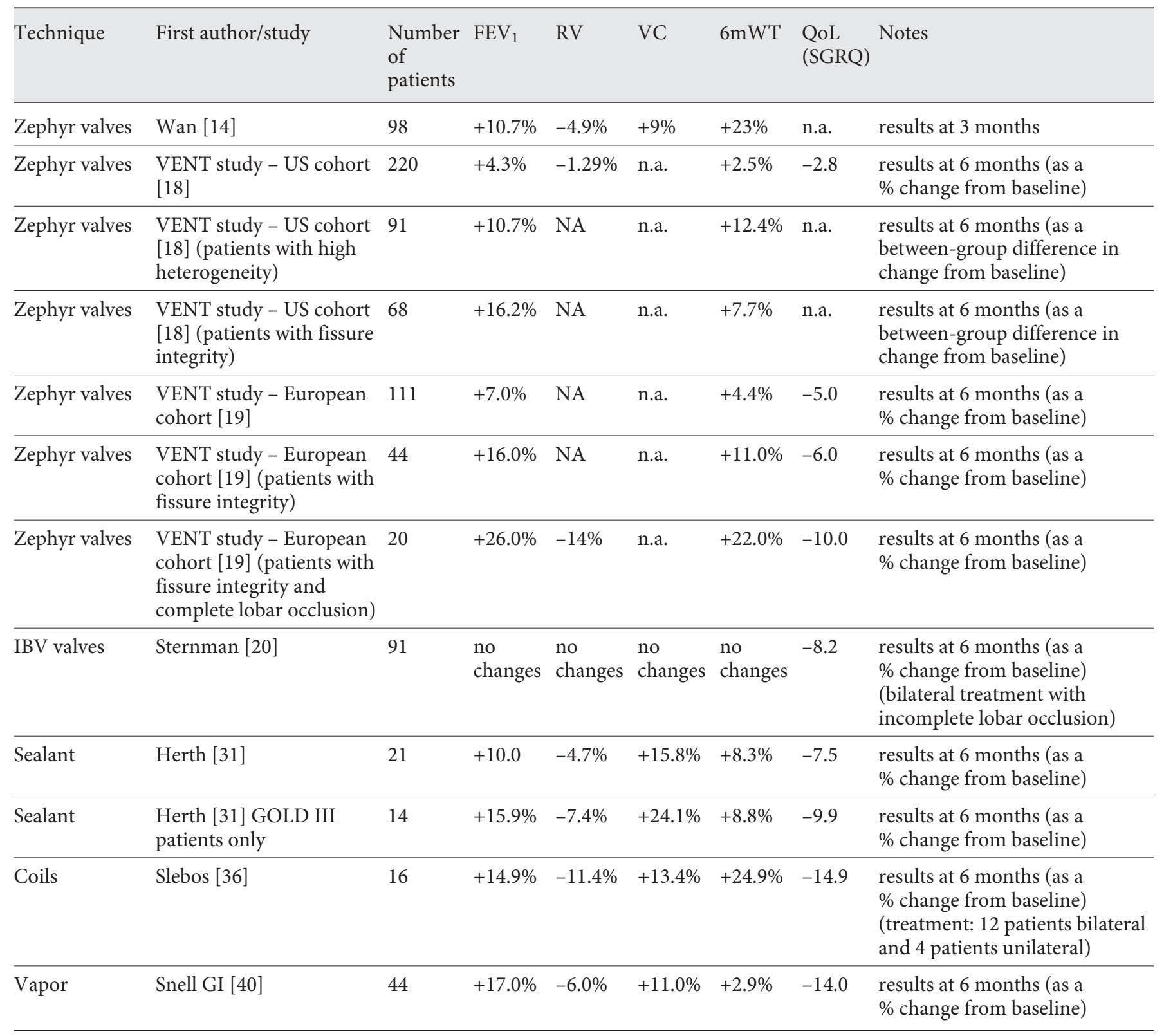

n.a. = Not available; QoL $=$ quality of life; $\mathrm{VC}=$ vital capacity.

pulmonary pathophysiologists and radiologists is an essential step in achieving this aim.

According to these considerations, the bronchoscopic treatment of emphysema should be performed in selected centers with expertise in various treatment modalities. Such centers should also have the expertise to carefully select subjects based on clinical, functional and imaging characteristics and have the ability to follow up the patients, providing alternative therapies in the case of bronchoscopic treatment failure.

The high cost of all these systems is another crucial point that underscores the need for careful patient selection to best identify those who will and will not benefit from these procedures. 
Figure 8 shows the flow chart for the bronchoscopic treatment of emphysema at our institution that we follow and propose for further evaluation. Symptomatic patients with heterogeneous emphysema and severe hyperinflation (RV greater than 150\% predicted) are evaluated for treatment. If there is evidence of absent collateral ventilation (fissure integrity on CT scan and/or by Chartis evaluation), unidirectional valves are considered the preferred option (because this treatment is fully reversible and has been demonstrated, even if only in a minority of patients, to provide a huge improvement in functional parameters and quality of life). If there is improvement, the valves are kept in place and the patient is followed up. If there is no improvement after 3 months, they are removed and other modalities of treatment are considered. If there is evidence of collateral ventilation (no fissure in- tegrity on CT scan and/or by Chartis evaluation), other treatment modalities are considered. If lung tissue is deemed adequate, we consider coils, sealant or vapor; if deemed inadequate, sealant or vapor are the first options.

In conclusion, it must be underlined that the evidence on the efficacy and safety of bronchoscopic emphysema treatment is still based mainly on studies with small groups of patients. However, several trials are ongoing and in the near future we will acquire more knowledge which should lead to better optimization of these procedures. Meanwhile, the bronchoscopic treatment of emphysema cannot yet be considered a standard of care and patients should be treated in the context of clinical trials or controlled registries, with well-defined programs of evaluation and follow-up.

\section{References}

1 Brantigan OC, Mueller EA, Kress MB: A surgical approach to pulmonary emphysema. Am Surg 1957;23:789-804.

-2 Brantigan OC, Kress MB, Mueller EA: The surgical approach to pulmonary emphysema. Dis Chest 1961;39:485-501.

- 3 National Emphysema Treatment Trial Research Group: A randomized trial comparing lung-volume-reduction surgery with medical therapy for severe emphysema. $\mathrm{N}$ Engl J Med 2003;348:2059-2073.

-4 McKenna RJ Jr, Fischel RJ, Brenner M, et al: Use of the Heimlich valve to shorten hospital stay after lung reduction surgery for emphysema. Ann Thorac Surg 1996;61:1115-1117.

5 Sanchez PG, Charles Kucharczuk J, Su S, et al: National Emphysema Treatment Trial redux: accentuating the positive. J Thorac Cardiovasc Surg 2010;140:564-572.

6 Ingenito EP, Reilly JJ, Mentzer SJ, et al: Bronchoscopic volume reduction: a safe and effective alternative to surgical therapy for emphysema. Am J Respir Crit Care Med 2001; 164:295-301.

7 Toma TP: The flexible bronchoscopic approach to lung volume reduction. Pneumologia 2001;2:97-100.

8 Watanabe Y, Matsuo K, Tamaoki A, et al: Bronchial occlusion with endobronchial Watanabe spigots. J Bronchol 2003;10:264267.

9 Toma TP, Matsuo K, Tamaoki A, et al: Endoscopic bronchial occlusion with spigots in patients with emphysema (abstract). Am J Respir Crit Care Med 2002;165:B9.
10 Miyazawa $\mathrm{H}$, Noto $\mathrm{H}$, Tniguchi $\mathrm{H}$, et al: Bronchoscopic lung volume reduction using silicone spigots in patients with severe emphysema. XIII World Congress for Bronchology, Barcelona, June 2004, abstract p 17.

11 Watanabe Y, Fujii H, Morichika D, et al: Bronchial occlusion with endoscopic Watanabe spigots on pulmonary diseases and bronchoscopic lung volume reduction. 16th World Congress for Bronchology, Budapest, June 2010, abstract p 66 .

12 Snell GI, Holsworth L, Borill ZL, et al: The potential for bonchoscopic lung volume reduction using bronchial prostheses: a pilot study. Chest 2003;124:1073-1080.

13 Toma TP, Hopkinson NS, Hiller J, et al: Bronchoscopic volume reduction with valve implants in patients with severe emphysema. Lancet 2003;361:931-933.

14 Wan IYP, Toma TP, Geddes DM, et al: Bronchoscopic lung volume reduction for endstage emphysema. Report on the first $98 \mathrm{pa}-$ tients. Chest 2006;129:518-526.

15 Mitnzer W: Collateral ventilation; in Crystal RG (ed): The Lung: Scientific Foundations. New York, Raven Press, 1991, pp 1053-1063.

16 Morrell NW, Wignall BK, Biggs T, et al: Collateral ventilation and gas exchange in emphysema. Am J Respir Crit Care Med 1994; 150:635-641.

17 Strange C, Herth FJ, Kovitz KL, et al: Design of the Endobronchial Valve for Emphysema Palliation Trial (VENT): a nonsurgical method of lung volume reduction. BMC Pulm Med 2007;7:10.

18 Sciurba FC, Ernst A, Herth FJF, et al: A randomized study of endobronchial valves for advanced emphysema. N Engl J Med 2010; 363:1233-1244.
19 Herth FJF, Noppen M, Valipour A, et al: Efficacy predictors of lung volume reduction with Zephyr valves in a European cohort. Eur Respir J 2012;39:1334-1342.

-20 Sternman DH, Mehta AC, Wood DE, et al: A multicenter pilot study of a bronchial valve for the treatment of severe emphysema. Respiration 2010;79:222-233.

21 Ninane V, Geltner C, Bezzi M, et al: Multicentre European study for the treatment of advanced emphysema with bronchial valves. Eur Respir J 2012;39:1319-1325.

22 Eberhardt R, Gompelmann D, Schuhmann $\mathrm{M}$, et al: Complete unilateral versus partial bilateral endoscopic lung volume reduction in patients with bilateral lung emphysema. Chest 2012, E-pub ahead of print.

23 Miyazawa H, Yamamoto Y, Shinno H, et al: Bronchoscopic lung volume reduction with new silicone valve in patients with advanced emphysema and giant bulla. 16th World Congress for Bronchology, Budapest, June 2010,abstract p 68 .

24 Galluccio G, Lucantoni G: Bronchoscopic lung volume reduction for pulmonary emphysema: preliminary experience with a new Novatech endobronchial silicone one-way valve. Interact CardioVasc Thorac Surg 2010;11:213-215

25 Mantri S, Macaraeg C, Shetty S, et al: Measurement of collateral flow in the lung with a dedicated endobronchial catheter system. J Bronchol Intervent Pulmonol 2009;16:141144 .

26 Gompelmann D, Eberhardt R, Michaud G, et al: Predicting atelectasis by assessment of collateral ventilation prior to endobronchial lung volume reduction: a feasibility study. Respiration 2010;80:419-425. 
27 Ingenito EP, Berger RL, Henderson AC, et al: Bronchoscopic lung volume reduction using tissue engineering principles. Am J Respir Crit Care Med 2003;167:771-778.

28 Pinto-Plata V, Reilly J, Rafaely Y, et al: Biologic lung volume reduction for advanced emphysema. Chest 2006, Meeting of the American College of Chest Physicians, Salt Lake City, October 2006.

29 Reilly J, Washko G, Pinto-Plata V, et al: Biological lung volume reduction. A new bronchoscopic therapy for advanced emphysema, Chest 2007;131:1108-1113.

30 Crinier GJ, Pinto-Plata V, Strange C, et al: Biologic lung volume reduction in advanced upper lobe emphysema: phase 2 results. Am J Respir Crit Care Med 2009;179:791-798

- 31 Herth FJF, Gompelmann D, Stanzel F, et al: Treatment of advanced emphysema with emphysematous lung sealant (AeriSeal). Respiration 2011;82:36-45.

32 http://clinicaltrials.gov/ct2/show/NCT 01449292.

33 Ost D, Ernst R, Maxfield F, et al: Evaluation of a bronchoscopically delivered non-valve implant that mechanically compresses diseased lung for the treatment of emphysema. European Respiratory Society Meeting, Berlin, October 2008, abstract 1588.
34 Herth F, Eberhardt R, Ernst A: Pilot study of an improved lung volume reduction coil for the treatment of emphysema. Am J Respir Crit Care Med 2009;179:A6160.

35 Herth FJF, Eberhard R, Gompelmann D, et al: Bronchoscopic lung volume reduction with a dedicated coil: a clinical pilot study. Ther Adv Respir Dis 2010;4:225-231.

36 Slebos DJ, Klooster K, Ernst A, et al: Bronchoscopic lung volume reduction coil treatment of patients with severe heterogeneous emphysema. Chest 2011, E-pub ahead of print.

37 Emery MJ, Eveland RL, Eveland K, et al: Am J Respir Crit Care Med 2010;182:1282-1291.

38 Emery MJ, Laurent L, Couetil DVM, et al: Lung volume reduction by bronchoscopic thermal vapor ablation. Chest 2007;132: 439 s.

39 Snell G, Hopkins P, Westall G, et al: A feasibility and safety study of bronchoscopic thermal vapor ablation: a novel emphysema therapy. Ann Thorac Surg 2009;88:19931998.

40 Snell G, Herth FJF, Hopkins P, et al: Bronchoscopic thermal vapor ablation therapy in the management of heterogeneous emphysema. ERJ 2012;39:1326-1333.
1 Gompelmann D, Heussel CP, Eberhardt R, et al: Efficacy of bronchoscopic thermal vapor ablation and lobar fissure completeness in patients with heterogeneous emphysema. Respiration 2012;83:400-406.

42 Rendina EA, De Giacomo T, Venuta F, et al: Feasibility and safety of the airway bypass procedure for patients with emphysema. J Thorac Cardiovasc Surg 2003;125:12941299.

43 Lausberg HF, Chino K, Patterson GA, et al: Bronchial fenestration improves expiratory flow in emphysematous human lung. Ann Thorac Surg 2003;75:393-397.

44 Choong CK, Macklem PT, Pierce JA, et al: Airway bypass improves the mechanical properties of explanted emphysematous lungs. Am J Respir Crit Care Med 2008; 178: 902-905.

45 Cardoso PF, Snell GI, Hopkins P, et al: Clinical application of airway bypass with paclitaxel-eluting stents: early results. J Thorac Cardiovasc Surg 2007;134:974-981

- 46 Shah PL, Slebos DJ, Cordoso PFG, et al: Bronchoscopic lung-volume reduction with Exhale airways stents for emphysema (EASE trial): randomised, sham controlled, multicentre trial. Lancet 2011;378:997-1005.

47 http://www.broncus.com/patients.asp. 